Hongjian Shi, Department of Mathematics, Simon Fraser University, B.C., Canada V5A 1S6 e-amil: hshi@@cs.sfu.ca

\title{
SOME TYPICAL PROPERTIES OF SYMMETRICALLY CONTINUOUS FUNCTIONS, SYMMETRIC FUNCTIONS AND CONTINUOUS FUNCTIONS
}

\begin{abstract}
In this paper we show that the typical symmetrically continuous function and the typical symmetric function have $c$-dense sets of points of discontinuity. Also we show the existence of a nowhere symmetrically differentiable function and a nowhere quasi-smooth function by showing directly such functions are typical in the space of all real continuous functions.
\end{abstract}

\section{Introduction}

A function $f: \mathbb{R} \rightarrow \mathbb{R}$ is said to be symmetrically continuous at $x \in \mathbb{R}$ if

$$
\lim _{h \rightarrow 0}[f(x+h)-f(x-h)]=0 .
$$

A function $f: \mathbb{R} \rightarrow \mathbb{R}$ is said to be symmetric at $x \in \mathbb{R}$ if

$$
\lim _{h \rightarrow 0}[f(x+h)+f(x-h)-2 f(x)]=0 .
$$

In 1964 Stein and Zygmund [1, p. 25] showed that if $f: \mathbb{R} \rightarrow \mathbb{R}$ is Lebesgue measurable and is symmetrically continuous on a Lebesgue measurable set $E$, then $f$ is continuous a.e. on $E$. Also they obtained the same conclusion for symmetric functions [1, p. 27]. In 1971 Preiss [1, p. 52] constructed a

Key Words: symmetrically continuous function, symmetric function, continuous function, nowhere symmetrically differentiable function, nowhere quasi-smooth function

Mathematical Reviews subject classification: Primary: 26A15, 26A27

Received by the editors December 6, 1994 
bounded measurable, $2 \pi$-periodic function that is symmetrically continuous everywhere and whose set of points of discontinuity is of power $c$. In 1989 Tran in [2] constructed a bounded measurable symmetric function whose set of points of discontinuity is of power $c$, and also showed that the absolute value function of this function is symmetrically continuous and its set of points of discontinuity is of power $c$.

In 1964 Neugebauer first studied typical properties of symmetric functions. He showed that the typical function of the set of all bounded, measurable symmetric functions equipped with supremum metric has a dense set of discontinuity. His methods would also give a typical result for symmetrically continuous functions.

In Section 2 by using the Preiss and Tran constructions we give an elementary proof to show that the typical symmetrically continuous function and the typical symmetric function have $c$-dense sets of points of discontinuity. This answers two questions posed in [1, p. 422].

Let us use the following expressions,

$$
\begin{aligned}
& D^{1} f(x, h)=[f(x+h)-f(x-h)] / h, \\
& D^{2} f(x, h)=[f(x+h)+f(x-h)-2 f(x)] / h .
\end{aligned}
$$

In 1969 Filipczak in [3] constructed a continuous function $f$ defined on $[0,1]$ which satisfies for each $x \in(0,1), \limsup _{h \rightarrow 0} D^{1} f(x, h)=+\infty$. In 1972 Kostyrko in [4] used this example to show that the typical function $f \in C[0,1]$, the set of all real continuous functions with the supremum metric, satisfies for each $x \in(0,1)$,

$$
\limsup _{h \rightarrow 0} D^{1} f(x, h)=+\infty \text { and } \liminf _{h \rightarrow 0} D^{1} f(x, h)=-\infty .
$$

In 1987 Evans [5, Theorem 1] constructed a function $f \in C[0,1]$ which satisfies that for each $x \in(0,1)$,

$$
\begin{gathered}
\underset{h \rightarrow 0^{+}}{\operatorname{ap} \limsup _{n}} D^{1} f(x, h)=+\infty, \quad \operatorname{ap} \liminf _{h \rightarrow 0^{+}} D^{1} f(x, h)=-\infty, \\
\text { and ap } \limsup _{h \rightarrow 0^{+}}\left|D^{2} f(x, h)\right|=+\infty .
\end{gathered}
$$

He used this example to show that such functions are typical in $C[0,1]$.

In Section 3 we directly show that the typical function $f \in C[0,1]$ satisfies for each $x \in(0,1)$,

$$
\limsup _{h \rightarrow 0}\left|D^{1} f(x, h)\right|=+\infty
$$

$$
\limsup _{h \rightarrow 0}\left|D^{2} f(x, h)\right|=+\infty
$$


without using the constructions of Filipczak and Evans.

Throughout this paper, $B S C[a, b]$ denotes the set of all bounded measurable, symmetrically continuous functions defined on the interval $[a, b]$ and equipped with the supremum metric $\rho$, and $B S[a, b]$ denotes the set of all bounded measurable, symmetric functions defined on $[a, b]$ and equipped with the supremum metric $\rho . D(f)$ denotes the set of points of discontinuity of function $f$. $A^{c}$ denotes the complement of a set $A$.

\section{Typical Properties of Symmetrically Continuous Func- tions and Symmetric Functions}

Lemma 1 (Tran [2 )]There are functions $g_{1} \in B S C[a, b]$ and $g_{2} \in B S[a, b]$ both of which have continuum points of discontinuity in every subinterval of $[a, b]$.

Proof. Tran gave a construction of a function $g \in B S[a, b]$ for which $D(g)$ is of power $c$ and constructed $g_{1}$ and $g_{2}$ from $g$. We can also use the Preiss result $\left[1\right.$, p. 52] to construct a function $g_{1}$ as in the lemma. Let $\left\{\left(a_{n}, b_{n}\right)\right\}$ be an enumeration of the set of all subintervals of $[a, b]$ with rational endpoints. For every $n$ there are a set $E_{n}$ that is of power $c$ and contained in $\left(a_{n}, b_{n}\right)$ and a symmetrically continuous function $f_{n}$ such that $0 \leq f_{n} \leq 1, f_{n}(x)>0$ for $x \in E_{n}$ and $f_{n}(x)=0$ outside of a set of measure zero. Note that such a function is discontinuous at a point if and only if it is positive there. Set

$$
g_{1}=\sum_{n=1}^{\infty} \frac{1}{2^{n}} f_{n}
$$

Then $g_{1}$ too is symmetrically continuous everywhere and is discontinuous precisely on the set $\left\{x \in[a, b]: g_{1}(x)>0\right\}$. Clearly this latter set is $c$-dense in $[a, b]$.

Theorem 2 Given $(c, d) \subseteq[a, b]$, let

$$
A((c, d))=\{f \in B S C[a, b]: D(f) \bigcap(c, d) \text { is of power } c\}
$$

Then $A((c, d))$ is a dense open set in $B S C[a, b]$.

Proof. Let $\left\{f_{n}\right\} \subseteq A((c, d))^{c}$ be a convergent sequence. Then there is a function $f \in B S C[a, b]$ such that $f_{n} \longrightarrow f$ uniformly. Let $e_{n}$ denote the set $D\left(f_{n}\right) \bigcap(c, d)$. Then $e_{n}$ is at most countable and so the union $\bigcup_{n=1}^{\infty} e_{n}$ is at 
most countable. We know that $f$ is continuous at each point $x \in(c, d) \backslash$ $\bigcup_{n=1}^{\infty} e_{n}$, so $f \in A((c, d))^{c}$. Hence $A((c, d))^{c}$ is closed and $A((c, d))$ is open.

Now we show that $A((c, d))$ is dense in $B S C[a, b]$. For every ball $B(f, \epsilon) \subseteq$ $B S C[a, b]$, if $f \in A((c, d))$ there is nothing to prove. We assume $f \in A((c, d))^{c}$. Then $f$ has at most countably many points of discontinuity in $(c, d)$. From Lemma 1 there is a function $g \in B S C[a, b]$ such that $g$ has a $c$-dense set of points of discontinuity on $(c, d)$. Let $M$ be a constant such that $|g(x)| \leq M$ for all $x \in[a, b]$ and set $h=f+\frac{\epsilon}{2 M} g$. Then $h \in B S C[a, b]$ is discontinuous in continuum many points of $(c, d)$ and

$$
\rho(h, f)=\rho\left(f+\frac{\epsilon}{2 M} g, f\right)=\rho\left(\frac{\epsilon}{2 M} g, 0\right)<\epsilon
$$

where $\rho$ is the supremum metric on $B S C[a, b]$. Thus $h \in A((c, d)) \bigcap B(f, \epsilon)$ and hence $A((c, d))$ is dense.

Theorem 3 The typical function $f \in B S C[a, b]$ has a c-dense set of points of discontinuity.

Proof. From Theorem $2 A(I)$ is a dense open set for each open subinterval $I$. The result follows by taking the intersection $\bigcap_{I} A(I)$ for all rational open subintervals $I \subseteq[a, b]$.

The same methods can be used to prove the following theorem.

Theorem 4 The typical function $f \in B S[a, b]$ has a c-dense set of points of discontinuity.

\section{An Application of the Baire Category Theorem to the Space of Continuous Functions}

Lemma 5 Let $f \in C[0,1], n$ be a positive integer, $m$ and $\epsilon$ be two given positive constants. Then there exists a finite piecewise linear function $g \in$ $C[0,1]$ such that for each $x \in[0,1],|f(x)-g(x)|<\epsilon$ and for each $x \in[1 / n, 1-$ $1 / n],\left|D^{2} g(x, h)\right|>m$ for some $h$ with $0<|h|<1 / n$.

Proof. The function $f$ is uniformly continuous on $[0,1]$. For $\epsilon>0$ there exists $\delta_{1}>0$ such that $\left|f\left(x_{1}\right)-f\left(x_{2}\right)\right|<\epsilon / 16$ whenever $x_{1}, x_{2} \in[0,1],\left|x_{1}-x_{2}\right|<\delta_{1}$. Take $\delta=\min \left\{\frac{\epsilon}{6 m}, \frac{\delta_{1}}{10}, \frac{1}{10 n}\right\}$ and partition $[0,1]$ as $0=x_{0}<x_{1}<\cdots<x_{k}=1$. Here $x_{i}-x_{i-1}=\delta$ if $i$ is not a number of the form $4 l+2$ where $l$ is a nonnegative integer. If $i$ is a number of the form $4 l+2, x_{i}-x_{i-1}=3 \delta$ except $k=4 l+2$. 
If $k$ is a number of form $4 l+2, x_{k}-x_{k-1}=\delta$ or $2 \delta$ or $3 \delta$ depending on how many subintervals we get if we partition $[0,1]$ into subintervals with length $\delta$.

Let $g$ be a finite piecewise linear function which connects the following points $a_{0}, a_{1}, a_{2}, \ldots, a_{k}$. Here $a_{0}=\left(x_{0}, f\left(x_{0}\right)+(3 / 8) \epsilon\right), a_{1}=\left(x_{1}, f\left(x_{1}\right)-\right.$ $(3 / 8) \epsilon)$. The point $a_{2}$ is the intersection point of the line $x=x_{2}$ with the half line starting from the point $a_{1}$ and parallel to the $x$-axis, $a_{3}=\left(x_{3}, f\left(x_{3}\right)+\right.$ $(3 / 8) \epsilon), a_{4}$ is the intersection point of the line $x=x_{4}$ with the half line starting from the point $a_{3}$ and parallel to the x-axis, $a_{5}=\left(x_{5}, f\left(x_{5}\right)-(3 / 8) \epsilon\right)$. Similarly as for $a_{2}$ we can define $a_{6}$, and continue in this way to get $a_{0}, a_{1}, a_{2}, \ldots, a_{k}$. See the figure (ii) where $r=\epsilon$.

We now verify that the function $g$ satisfies our requirements. Obviously $g$ is a finite piecewise linear, continuous function and for each $x \in[0,1]$, $|f(x)-g(x)|<\epsilon$. For the remainder we need to verify that for each $x \in$ $\left[x_{i-2}, x_{i+2}\right]$ as indicated in the figure (ii), $\left|D^{2} g(x, h)\right|>m$ for some $h$ with $0<|h|<1 / n$. We can assume $3<i<k-3$ since $x \in[1 / n, 1-1 / n]$ and $\delta \leq \frac{1}{10 n}$. For $x \in\left[x_{i-1}, x_{i}\right]$, choose $h=\min \left\{x-x_{i-2}, x_{i+1}-x\right\}$ and note $\delta \leq \frac{\epsilon}{6 m}$,

$$
\begin{aligned}
\left|D^{2} g(x, h)\right| & =\left|\frac{g(x+h)-g(x)}{h}\right|+\left|\frac{g(x)-g(x-h)}{h}\right| \\
& \geq \frac{(3 / 4) \epsilon-(1 / 16) \epsilon}{(5 / 2) \delta}=\frac{11 \epsilon}{40 \delta}>m .
\end{aligned}
$$

Partition $\left[x_{i}, x_{i+1}\right]$ into three subintervals of equal length $\left[x_{i}, x^{1}\right],\left[x^{1}, x^{2}\right]$ and $\left[x^{2}, x_{i+1}\right]$. For $x \in\left[x_{i}, x^{1}\right]$, choose $h=x_{i+1}-x$. Then

$$
\begin{aligned}
\left|D^{2} g(x, h)\right| & =\left|\frac{g(x+h)-g(x)}{h}\right|-\left|\frac{g(x)-g(x-h)}{h}\right| \\
& \geq(1-1 / 3) \frac{(3 / 4) \epsilon-(1 / 16) \epsilon}{\delta}=\frac{11 \epsilon}{24 \delta}>m .
\end{aligned}
$$

For $x \in\left[x^{1}, x^{2}\right]$, choose $h=x_{i+3}-x$. Then

$$
\begin{aligned}
\left|D^{2} g(x, h)\right| & =\left|\frac{g(x+h)-g(x)}{h}\right|+\left|\frac{g(x)-g(x-h)}{h}\right| \\
& \geq 2\left[\frac{(1 / 3)((3 / 4) \epsilon-(1 / 16) \epsilon)}{(2+(2 / 3)) \delta}\right]=\frac{11 \epsilon}{64 \delta}>m
\end{aligned}
$$


For $x \in\left[x^{2}, x_{i+1}\right]$, choose $h=x-x_{i}$. Then

$$
\begin{aligned}
\left|D^{2} g(x, h)\right| & =|| \frac{g(x-h)-g(x)}{h}|-|\left|\frac{g(x+h)-g(x)}{h}\right| \\
& \geq(1-1 / 3) \frac{(3 / 4) \epsilon-(1 / 16) \epsilon}{\delta}>m .
\end{aligned}
$$

For $x \in\left[x_{i+1}, x_{i+2}\right]$, choose $h=\min \left\{x-x_{i}, x_{i+3}-x\right\}$. Then

$\left|D^{2} g(x, h)\right|=|| \frac{g(x+h)-g(x)}{h}|+|\left|\frac{g(x-h)-g(x)}{h}\right| \geq \frac{(3 / 4) \epsilon-(1 / 16) \epsilon}{2 \delta}=\frac{11 \epsilon}{32 \delta}>m$.

For $x \in\left[x_{i-2}, x_{i-1}\right]$ using the same method for $x \in\left[x_{i}, x_{i+1}\right]$ we can show that the function $g$ satisfies our requirements. Hence the lemma follows.
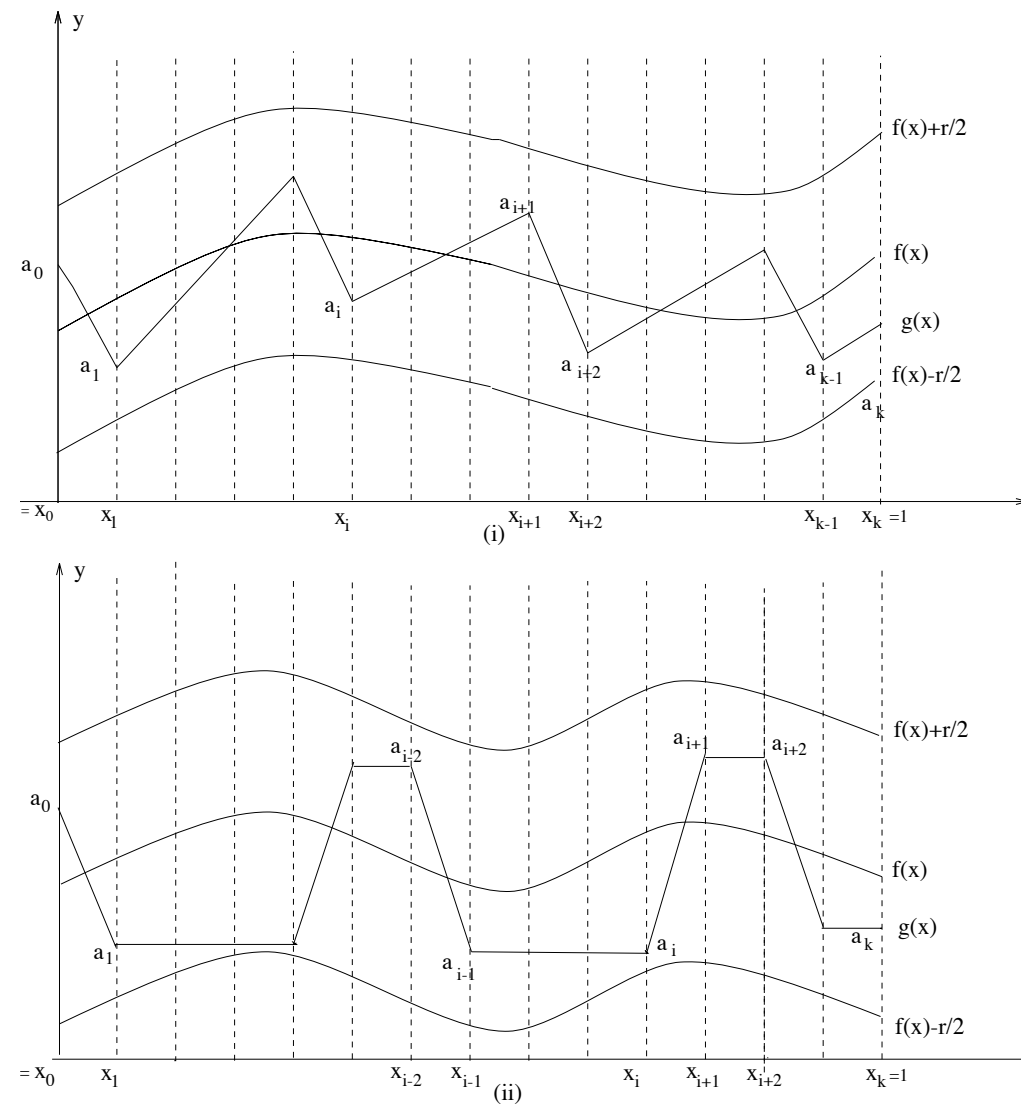
Theorem 6 The typical function $f \in C[0,1]$ satisfies (2) for all $x \in(0,1)$.

Proof. Let

$$
\begin{aligned}
& A=\left\{f \in C[0,1]: \begin{array}{l}
\text { there exist some point } x \in(0,1) \text { and constant } C \\
\text { such that } \limsup _{h \rightarrow 0}\left|D^{2} f(x, h)\right| \leq C
\end{array}\right\}, \\
& A_{n m}=\left\{f \in C[0,1]: \begin{array}{l}
\text { there exists some } x \in[1 / n, 1-1 / n] \text { such that } \\
\left|D^{2} f(x, h)\right| \leq m \text { whenever } 0<|h|<1 / n,
\end{array}\right\}
\end{aligned}
$$

Then $A=\bigcup_{n, m=1}^{\infty} A_{n m}$. Using the same standard arguments as in Theorem 2 and Lemma 5 we can show that each $A_{n m}$ is an open dense set in $C[0,1]$ and therefore the theorem follows.

Note that the analogous statement to Lemma 5 but using $D^{1}$ in place of $D^{2}$ is easier to prove and can be obtained by choosing a saw-tooth function with suitable slopes as in figure (i). Similar methods can be used to prove the following theorem.

Theorem 7 The typical function $f \in C[0,1]$ satisfies (1) for all $x \in(0,1)$.

Acknowledgment: Here I would like to express my sincere thanks to my supervisor Dr. Thomson for his guidance and support.

\section{References}

[1] Brian S. Thomson, Symmetric Properties of Real Functions, Marcel Dekker Inc., New York, 1994.

[2] T. C. Tran, Symmetric functions whose set of points of discontinuity is uncountable, Real Anal. Exch. 12 (1986-87), 496-509.

[3] L. Filipczak, Example d'un fonction continue privée de dérivée symétrique partout, Colloquium Mathematicum 20 (1969), 249-253.

[4] P. Kostyrko, On the symmetric derivative, Colloquium Mathematicum 25 (1972), 265-267.

[5] Michael J. Evans, Approximate smoothness of continuous functions, Colloquium Mathematicum 54 (1987), 307-313. 